\title{
Kerstersia gyiorum
}

National Cancer Institute

\section{Source}

National Cancer Institute. Kerstersia gyiorum. NCI Thesaurus. Code C127722.

A species of Gram-negative, catalase-positive, oxidase-negative bacteria in the in phylum

Proteobacteria that is positive for assimilation of caprylate and the absence of assimilation of isobutyrate, isovalerate, or glutarate. 